https://helda.helsinki.fi

\title{
Pointwise estimates to the modified Riesz potential
}

\section{Harjulehto, Petteri}

2018-07

Harjulehto , P \& Hurri-Syrjänen, R 2018 , ' Pointwise estimates to the modified Riesz

potential ' , Manuscripta Mathematica , vol. 156 , no. 3-4 , pp. 521-543 . https://doi.org/10.1007/s00229-017-0983-y

http://hdl.handle.net/10138/307540

https://doi.org/10.1007/s00229-017-0983-y

unspecified

acceptedVersion

Downloaded from Helda, University of Helsinki institutional repository.

This is an electronic reprint of the original article.

This reprint may differ from the original in pagination and typographic detail.

Please cite the original version. 


\title{
POINTWISE ESTIMATES TO THE MODIFIED RIESZ POTENTIAL
}

\author{
PETTERI HARJULEHTO AND RITVA HURRI-SYRJÄNEN
}

\begin{abstract}
In a smooth domain a function can be estimated pointwise by the classical Riesz potential of its gradient. Combining this estimate with the boundedness of the classical Riesz potential yields the optimal Sobolev-Poincaré inequality. We show that this method gives a Sobolev-Poincaré inequality also for irregular domains whenever we use the modified Riesz potential which arise naturally from the geometry of the domain. The exponent of the Sobolev-Poincaré inequality depends on the domain. The SobolevPoincaré inequality given by this approach is not sharp for irregular domains, although the embedding for the modified Riesz potential is optimal. In order to obtain the results we prove a new pointwise estimate for the Hardy-Littlewood maximal operator.
\end{abstract}

\section{INTRODUCTION}

In irregular domains a function can be estimated pointwise by a modified Riesz potential of its gradient where the Riesz potential depends on the geometry of the domain. When the modified Riesz potential is bounded from $L^{p}$ to $L^{H}$, here $L^{H}$ is an Orlicz space, this leads to the Poincaré inequality

$$
\left\|u-u_{D}\right\|_{L^{H}(D)} \leq C\|\nabla u\|_{L^{p}(D)} .
$$

This method is well known for smooth domains, or more precisely for John domains, and it gives the optimal Sobolev embedding, we refer to [5, Chapter 7] and [24]. We will show that this method does not give the optimal integrability in more irregular domains than John domains although the embedding for the Riesz potential is sharp.

We assume that $\varphi$ is a continuous, strictly increasing function on $[0, \infty)$ such that for some constant $C_{\varphi}$

$$
\frac{\varphi\left(t_{1}\right)}{t_{1}} \leq C_{\varphi} \frac{\varphi\left(t_{2}\right)}{t_{2}} \quad \text { whenever } \quad 0<t_{1} \leq t_{2}
$$

Date: September 22, 2017.

2010 Mathematics Subject Classification. 31C15 , 42B20, 42B25, 26D10, 46E30.

Key words and phrases. Riesz potential, Hardy-Littlewood maximal operator, pointwise estimate, Orlicz space, Sobolev-Poincaré inequality, non-smooth domain. 
In Theorem 4.4 we show that if $D$ is a $\varphi$-John domain, Definition 4.1, then

$$
\left|u(x)-u_{D}\right| \leq C \int_{D} \frac{|\nabla u(y)|}{\varphi(|x-y|)^{n-1}} d y
$$

for every $u \in L_{1}^{1}(D)$. Here $u_{D}$ is the integral average of $u$ over $D$. For $\varphi(t)=t$ this result was proved in [5, Chapter 7] and [24].

F.-Y. Maeda, Y. Mizuta, T. Ohno, and T. Shimomura showed in [16, Corollary 6.5] that the modified Riesz potential is bounded from $L^{p}$ to $L^{H}$, where $L^{H}$ is an Orlicz space and the Orlicz function $H$ depends on $\varphi$. We give an alternative proof for their result in Theorem 3.8. Our proof is based on a new pointwise estimate by the Hardy-Littlewood maximal operator, see Theorem 3.5.

These results together give the Sobolev-Poincaré type inequality

$$
\left\|u-u_{D}\right\|_{L^{H}(D)} \leq C\|\nabla u\|_{L^{p}(D)}
$$

that is presented in Theorem 5.2. As a special case we study the situation

$$
\varphi(t)=\frac{t^{\alpha}}{\log ^{\beta}\left(e+t^{-1}\right)} \quad \text { and } \quad H(t)=\left(\frac{t}{\log ^{\beta(n-1)}(m+t)}\right)^{\frac{n p}{\alpha p(n-1)+n(1-p)}},
$$

where $1 \leq \alpha<n /(n-1)$ and $\beta \geq 0$. This special case has been earlier studied by the authors in [10]. This is sharp when $\alpha=1$ and $\beta=0$ or when $\alpha>1, \beta=0$ and $p=1$. However, when $\alpha>1, \beta=0$ and $p>1$ the Sobolev-Poincaré type inequality $\left\|u-u_{D}\right\|_{L^{H}(D)} \leq C\|\nabla u\|_{L^{p}(D)}$ is not sharp. We conjecture that the right exponent is $\frac{n p}{\alpha(n-1)-p+1}$ also in the case $\beta>0$. For more details and for references we refer to the discussion after Theorem 5.2. Somewhat suprisingly, in Theorem 6.5 we show that in these cases the Riesz embedding $L^{p} \hookrightarrow L^{H}$ is sharp in the sense that the exponent $\frac{n p}{\alpha p(n-1)+n(1-p)}$ cannot be larger and the exponent $\beta(n-1)$ cannot be smaller. Our conclusion is that in very irregular domains the natural modified Riesz potential is not the right tool for the optimal Sobolev-Poincaré type inequalities.

The paper is organised as follows. We have collected main properties of Orlicz spaces to Section 2. In Section 3 we prove pointwise estimates to the modified Riesz potential and prove boundedness. In Section 4 we show that our modified Riesz potential arises naturally from the geometry of the domain. In Section 5 we prove the Sobolev-Poincaré inequalities. In Section 6 we study the sharpness of the results.

\section{Preliminaries}

Throughout this paper we assume that the function $H:[0, \infty) \rightarrow$ $[0, \infty)$ has the properties: (1) $H$ is continuous; (2) $H$ is strictly increasing; (3) $H$ is convex; (4) $\lim _{t \rightarrow 0^{+}} \frac{H(t)}{t}=0$ and $\lim _{t \rightarrow \infty} \frac{H(t)}{t}=\infty$; (5) $\frac{H(t)}{t}<\frac{H(s)}{s}$ for $0<t<s$; and (6) there exists $C_{H}^{\Delta_{2}}$ such that 
$H(2 t) \leq C_{H}^{\Delta_{2}} H(t)$ for all $t>0$. In other words, we suppose that $H$ is an $N$-function, $[1,8.2]$, that satisfies the $\Delta_{2}$-condition.

Let $G$ in $\mathbb{R}^{n}$ be an open set. The Orlicz class is a set of all measurable functions $u$ defined on $G$ such that

$$
\int_{G} H(|u(x)|) d x<\infty .
$$

We study the Orlicz space $L^{H}(G)$ which means a space of all measurable functions $u$ defined on $G$ such that

$$
\int_{G} H(\lambda|u(x)|) d x<\infty
$$

for some $\lambda>0$.

When the function $H$ satisfies the $\Delta_{2}$-condition, then the space $L^{H}(G)$ is a vector space and it is equivalent to the corresponding Orlicz class. We study these Orlicz spaces and call their functions Orlicz functions. The Orlicz space $L^{H}(G)$ equipped with the Luxemburg norm

$$
\|u\|_{L^{\Phi}(G)}=\inf \left\{\lambda>0: \int_{G} \Phi\left(\frac{|u(x)|}{\lambda}\right) d x \leq 1\right\}
$$

is a Banach space.

We note that if the $n$-Lebesque measure of $G$ is finite, then there is a continuous embedding $L^{H}(G) \hookrightarrow L^{1}(G)$. For more information about Orlicz spaces we refer to [1, Section 8] and [6, Section 6.3].

The space of locally integrable functions defined on an open set $G$ is written as $L_{\text {loc }}^{1}(G)$. We recall that the space $L_{p}^{1}(G), 1 \leq p<\infty$, is a space of distributions on $G$ with the first order derivatives in the space $L^{p}(G)$.

An open ball with a center $x$ and radius $r>0$ is written as $B(x, r)$. The corresponding closed ball is denoted by $\bar{B}(x, r)$. Given any proper subset $A$ of $\mathbb{R}^{n}$ and any $x \in \mathbb{R}^{n}$, the distance between $x$ and the boundary $\partial A$ is written as $\operatorname{dist}(x, \partial A)$, and $\operatorname{diam}(A)$ stands for the diameter of $A$. The characteristic function of a set $A$ is denoted by $\chi_{A}$. When $A$ in $\mathbb{R}^{n}$ is a Lebesgue measurable set with positive $n$-Lebesgue measure $|A|$ we write the integral average of an integrable function $u$ in $A$ as

$$
u_{A}=f_{A} u(x) d x=|A|^{-1} \int_{A} u(x) d x .
$$

We let $C(*, \cdots, *)$ and $C_{*}$ denote constants which depend on the given quantities only. In the calculations from one line to the next line we usually write $C$ for constants when it is not important to specify constants' dependence on the quantities appearing in the calculations. From line to line $C$ might stand for a different constant. 


\section{Pointwise estimates for a modified Riesz potential}

The classical centered Hardy-Littlewood maximal function is written as

$$
M f(x)=\sup _{r>0} f_{B(x, r)}|f(y)| d y
$$

where $f$ is a locally integrable function defined on $\mathbb{R}^{n}$, [25, Section 1]. We give two pointwise estimates by using the Hardy-Littlewood maximal operator in Lemmas 3.1 and 3.3. Lars Inge Hedberg stated and proved the corresponding results when $\varphi(t)=t$, [13, Lemma (a), (b)]. A. Cianchi and B. Stroffolini used the Hedberg method for the classical Riesz potential when functions are Orlicz functions, [4, Theorem 1 , Corollary 1].

3.1. Lemma. Let $\varphi:[0, \infty) \rightarrow[0, \infty)$ be a continuous, strictly increasing function. Let $h:[0, \infty) \rightarrow[0, \infty)$ satisfy

$$
\sum_{k=1}^{\infty} \frac{\left(2^{-k} t\right)^{n}}{\varphi\left(t 2^{-k}\right)^{n-1}} \leq h(t) \quad \text { for all } \quad t>0 .
$$

Let $\delta>0$ be given. If $f \in L_{\text {loc }}^{1}\left(\mathbb{R}^{n}\right)$, then there exists a constant $C(n)$ such that the inequality

$$
\int_{B(x, \delta)} \frac{|f(y)|}{\varphi(|x-y|)^{n-1}} d y \leq C(n) h(\delta) M f(x)
$$

holds for every $x \in \mathbb{R}^{n}$.

Proof. Let $x \in \mathbb{R}^{n}$ be fixed and let $\delta$ be given. Let us divide the ball $B(x, \delta)$ into annuli. By bringing in the Hardy-Littlewood maximal operator and by using inequality (3.2) we obtain

$$
\begin{aligned}
\int_{B(x, \delta)} \frac{|f(y)|}{\varphi(|x-y|)^{n-1}} d y & \leq \sum_{k=1}^{\infty} \varphi\left(\delta 2^{-k}\right)^{1-n} \int_{\left\{z: 2^{-k} \delta \leq|x-z|<2^{-k+1} \delta\right\}}|f(y)| d y \\
& \leq C(n) \sum_{k=1}^{\infty} \frac{\left(2^{-k} \delta\right)^{n}}{\varphi\left(\delta 2^{-k}\right)^{n-1}} f_{\left\{z:|x-z|<2^{-k+1} \delta\right\}}|f(y)| d y \\
& \leq C(n) M f(x) \sum_{k=1}^{\infty} \frac{\left(2^{-k} \delta\right)^{n}}{\varphi\left(\delta 2^{-k}\right)^{n-1}} \leq C(n) M f(x) h(\delta) .
\end{aligned}
$$

We consider the integral over the set $\mathbb{R}^{n} \backslash B(x, \delta)$, too.

3.3. Lemma. Let $\varphi:[0, \infty) \rightarrow[0, \infty)$ be a continuous strictly increasing function such that inequality (1.1) holds. Let $1 \leq p<n$. Let $\delta>0$ be given. If $\|f\|_{L^{p}\left(\mathbb{R}^{n}\right)} \leq 1$, then there is a constant $C$, depending on $n, p$, and $C_{\varphi}$ only such that the inequality

$$
\int_{\mathbb{R}^{n} \backslash B(x, \delta)} \frac{|f(y)|}{\varphi(|x-y|)^{n-1}} d y \leq C \varphi(\delta)^{1-n} \delta^{n\left(1-\frac{1}{p}\right)}
$$


holds for every $x \in \mathbb{R}^{n}$.

The inequality in Lemma 3.3 has been proved for the function $\varphi(t)=$ $t^{\alpha} / \log ^{\beta}\left(e+t^{-1}\right)$ when $1 \leq \alpha<1+1 /(n-1)$ and $\beta \geq 0$ in [10, Lemma 3.2]. The proof here is a generalization of this earlier result. We give the proof for the sake of completeness.

Proof of Lemma 3.3. Suppose that $1<p<n$ and let us write $p^{\prime}=$ $p /(p-1)$. Let the point $x \in \mathbb{R}^{n}$ be fixed and let $\delta>0$ be given. By Hölder's inequality we obtain

$$
\begin{aligned}
\int_{\mathbb{R}^{n} \backslash B(x, \delta)} \frac{|f(y)|}{\varphi(|x-y|)^{n-1}} d y & \leq\|f\|_{L^{p}\left(\mathbb{R}^{n}\right)}\left\|\chi_{\mathbb{R}^{n} \backslash B(x, \delta)} \varphi(|x-\cdot|)^{1-n}\right\|_{L^{p^{\prime}\left(\mathbb{R}^{n}\right)}} \\
& \leq\left\|\chi_{\mathbb{R}^{n} \backslash B(x, \delta)} \varphi(|x-\cdot|)^{-n}\right\|_{L^{(n-1) p^{\prime} / n}\left(\mathbb{R}^{n}\right)}^{(n-1) / n}
\end{aligned}
$$

For every $y \in \mathbb{R}^{n} \backslash B(x, \delta)$,

$$
\begin{aligned}
\varphi(|x-y|)^{-n} & =C(n)|B(y, \varphi(|x-y|))|^{-1} \\
& =C(n) \int_{B(y, 2|x-y|)} \chi_{B(x, \delta)}(z)|B(x, \delta)|^{-1} \frac{|B(y, 2|x-y|)|}{|B(y, \varphi(|x-y|))|} d z .
\end{aligned}
$$

By assumption (1.1) we obtain that

$$
\frac{|B(y, 2 t)|}{|B(y, \varphi(t))|} \leq C\left(n, C_{\varphi}\right)\left(\frac{\delta}{\varphi(\delta)}\right)^{n}
$$

for every $t \geq \delta$. Hence,

$$
\varphi(|x-y|)^{-n} \leq C\left(n, C_{\varphi}\right)\left(\frac{\delta}{\varphi(\delta)}\right)^{n} M\left(\chi_{B(x, \delta)}|B(x, \delta)|^{-1}\right)(y)
$$

for every $y \in \mathbb{R}^{n} \backslash B(x, \delta)$.

Since $1<p<n$, we have $1<\frac{n-1}{n} p^{\prime}<\infty$. Thus, the Hardy-Littlewood maximal operator is bounded in $L^{(n-1) p^{\prime} / n}\left(\mathbb{R}^{n}\right)$, [25, Section 1 , Theorem $1(\mathrm{c})]$, and we obtain

$$
\begin{aligned}
& \left\|\chi_{\mathbb{R}^{n} \backslash B(x, \delta)} \varphi(|x-\cdot|)^{-n}\right\|_{L^{(n-1) p^{\prime} / n}\left(\mathbb{R}^{n}\right)}^{(n-1) / n} \\
& \quad \leq C\left(n, C_{\varphi}\right)\left(\frac{\delta}{\varphi(\delta)}\right)^{n-1}\left\|M\left(\chi_{B(x, \delta)}|B(x, \delta)|^{-1}\right)\right\|_{L^{(n-1) p^{\prime} / n}\left(\mathbb{R}^{n}\right)}^{(n-1) / n} \\
& \quad \leq C\left(n, C_{\varphi}, p\right)\left(\frac{\delta}{\varphi(\delta)}\right)^{n-1}\left\|\chi_{B(x, \delta)}|B(x, \delta)|^{-1}\right\|_{L^{(n-1) p^{\prime} / n}\left(\mathbb{R}^{n}\right)}^{(n-1) / n} \\
& \quad \leq C\left(n, C_{\varphi}, p\right) \varphi(\delta)^{1-n}\left\|\chi_{B(x, \delta)}\right\|_{L^{(n-1) p^{\prime} / n\left(\mathbb{R}^{n}\right)}}^{(n-1) / n} \leq C\left(n, C_{\varphi}, p\right) \varphi(\delta)^{1-n} \delta^{\frac{n}{p^{\prime}}} .
\end{aligned}
$$

Hence, the claim is proved whenever $1<p<\infty$.

If $p=1$, and $\delta>0$ is given, and $\|f\|_{L^{1}\left(\mathbb{R}^{n}\right)} \leq 1$, then

$$
\int_{\mathbb{R}^{n} \backslash B(x, \delta)} \frac{|f(y)|}{\varphi(|x-y|)^{n-1}} d y \leq \varphi(\delta)^{1-n} \int_{\mathbb{R}^{n} \backslash B(x, \delta)}|f(y)| d y \leq \varphi(\delta)^{1-n} .
$$


3.4. Remark. Let us assume that $\varphi(t)=\frac{t^{\alpha}}{\log ^{\beta}\left(e+t^{-1}\right)}, \alpha \in\left[1,1+\frac{1}{n-1}\right)$ and $\beta \geq 0$. We use this $\varphi$ in Corollary 5.10. In this case the restriction $p<n$ in Lemma 3.3 can be replaced by the inequality $p<n /(n-\alpha(n-1))$. This yields that in Theorems 3.5, 3.8 and 5.1 the restriction $p<n$ can be replaced by $p<n /(n-\alpha(n-1))$. In the proof of Lemma 3.3 we may estimate the term $\left\|\chi_{\mathbb{R}^{n} \backslash B(x, \delta)} \varphi(|x-\cdot|)^{1-n}\right\|_{L^{p^{\prime}\left(\mathbb{R}^{n}\right)}}$ by using the following calculation:

$$
\begin{aligned}
& \left\|\chi_{\mathbb{R}^{n} \backslash B(x, \delta)} \varphi(|x-y|)^{1-n}\right\|_{L^{p^{\prime}\left(\mathbb{R}^{n}\right)}}=\left(\int_{\mathbb{R}^{n} \backslash B(x, \delta)} \varphi(|x-\cdot|)^{p^{\prime}(1-n)} d y\right)^{\frac{1}{p^{\prime}}} \\
& \quad=\left(C(n) \int_{\delta}^{\infty} \varphi(t)^{p^{\prime}(1-n)} t^{n-1} d t\right)^{\frac{1}{p^{\prime}}} \\
& \quad=C(n, p)\left(\int_{\delta}^{\infty} t^{\alpha p^{\prime}(1-n)+n-1} \log ^{\beta p^{\prime}(n-1)}\left(e+t^{-1}\right) d t\right)^{\frac{1}{p^{\prime}}} \\
& \quad \leq C(n, p) \log ^{\beta(n-1)}\left(e+\delta^{-1}\right)\left(\int_{\delta}^{\infty} t^{\alpha p^{\prime}(1-n)+n-1} d t\right)^{\frac{1}{p^{\prime}}} .
\end{aligned}
$$

The last integral is finite if $\alpha p^{\prime}(1-n)+n<0$. In this case we obtain

$$
\begin{aligned}
\left\|\chi_{\mathbb{R}^{n} \backslash B(x, \delta)} \varphi(|x-y|)^{1-n}\right\|_{L^{p^{\prime}\left(\mathbb{R}^{n}\right)}} & \leq C(n, p) \log ^{\beta(n-1)}\left(e+\delta^{-1}\right) \delta^{\alpha(1-n)+\frac{n}{p^{\prime}}} \\
& =\varphi(\delta)^{1-n} \delta^{n\left(1-\frac{1}{p}\right)} .
\end{aligned}
$$

3.5. Theorem. Let $1 \leq p<n$ be given. Let $\varphi:[0, \infty) \rightarrow[0, \infty)$ be a continuous, strictly increasing function which satisfies condition (1.1). Suppose that there exists a continuous function $h:[0, \infty) \rightarrow[0, \infty)$ so that $(3.2)$ holds. Let $\delta:(0, \infty) \rightarrow[0, \infty)$ be a continuous function and let $H:[0, \infty) \rightarrow[0, \infty)$ be an Orlicz function satisfying the $\Delta_{2}$-condition. Suppose that there exists a finite constant $C_{H}$ such that the inequality

$$
H\left(h(\delta(t)) t+\varphi(\delta(t))^{1-n}(\delta(t))^{n\left(1-\frac{1}{p}\right)}\right) \leq C_{H} t^{p}
$$

holds for all $t>0$. Let $G$ in $\mathbb{R}^{n}$ be an open set. If $\|f\|_{L^{p}\left(\mathbb{R}^{n}\right)} \leq 1$, then there exists a constant $C$ such that the inequality

$$
H\left(\int_{G} \frac{|f(y)|}{\varphi(|x-y|)^{n-1}} d y\right) \leq C(M f(x))^{p}
$$

holds for every $x \in \mathbb{R}^{n}$. Here the constant $C$ depends on $n, p, C_{\varphi}, C_{H}$, and the $\Delta_{2}$-constant of $H$ only.

Proof. We may assume that $M f(x)>0$, since otherwise $f(x)=0$ almost everywhere. By Lemmas 3.1 and 3.3 there exists a constant $C$ such that we obtain

$$
\begin{aligned}
& \int_{G} \frac{|f(y)|}{\varphi(|x-y|)^{n-1}} d y \leq \int_{\mathbb{R}^{n}} \frac{|f(y)|}{\varphi(|x-y|)^{n-1}} d y \\
& \quad \leq C h(\delta(M f(x))) M f(x)+C \varphi(\delta(M f(x)))^{1-n}(M f(x))^{n\left(1-\frac{1}{p}\right)}
\end{aligned}
$$


for every $x$ in $\mathbb{R}^{n}$. Condition (3.6) implies for all $x$ in $\mathbb{R}^{n}$

$$
H\left(\int_{D} \frac{|f(y)|}{\varphi(|x-y|)^{n-1}} d y\right) \leq C(M f(x))^{p} .
$$

F.-Y. Maeda, Y. Mizuta, T. Ohno, and T. Shimomura showed in [16, Corollary 6.5] that the modified Riesz potential is bounded in generalized Orlicz spaces. The following result is a special case of their result. We have written the result for an open set $G$ in $\mathbb{R}^{n}$ to make it clear that the result does not depend on the geometry of the domain. This formulation also is more convenient when we connect it to irregular domains. We point out that it is possible to choose $G=\mathbb{R}^{n}$.

3.8. Theorem (Corollary 6.5 in [16]). Let $H$ be an Orlicz function and $\varphi$ be an increasing function as in Theorem 3.5. Let $G$ be an open set in $\mathbb{R}^{n}$. Then there exists a constant $C$ such that the inequality

$$
\int_{G} H\left(\int_{G} \frac{|f(y)|}{\varphi(|x-y|)^{n-1}} d y\right) d x \leq C
$$

holds for every $f$ when $\|f\|_{L \log L(G)} \leq 1$ if $p=1$ in (3.6), and for every $f$ when $\|f\|_{L^{p}(G)} \leq 1$ if $1<p<n$ in (3.6). Here the constant $C$ depends on $n, p, C_{\varphi}, C_{H}$, and the $\Delta_{2}$-constant of $H$ only.

We give an alternative proof for this theorem based on the Hedberg method.

Proof. Suppose that $1<p<n$. Let us assume that $\|f\|_{L^{p}(G)} \leq 1$. Then by Theorem 3.5 the inequality

$$
H\left(\int_{G} \frac{|f(y)|}{\varphi(|x-y|)^{n-1}} d y\right) \leq C(M f(x))^{p}
$$

holds for every $x \in G$. Since the Hardy-Littlewood maximal operator $M: L^{p} \rightarrow L^{p}$ is bounded whenever $1<p<\infty$, we obtain by integrating both sides of this inequality over $G$

$$
\int_{G} H\left(\int_{G} \frac{|f(y)|}{\varphi(|x-y|)^{n-1}} d y\right) d x \leq C \int_{G}(M f(x))^{p} d x \leq C \int_{G}|f(x)|^{p} d x \leq C .
$$

The proof in the case $p=1$ follows in the same lines; but the fact that the maximal operator $M: L \log L \rightarrow L^{1}$ is bounded had to be used instead of the boundedness of the maximal operator $M: L^{p} \rightarrow L^{p}$ whenever $1<p<\infty$.

3.9. Remark. Note that if the inequality

$$
\int_{G} H\left(\int_{G} \frac{|f(y)|}{\varphi(|x-y|)^{n-1}} d y\right) d x \leq C
$$

holds for every $f$ whenever $\|f\|_{L^{p}(G)} \leq 1$, where $1<p<\infty$, then

$$
\left\|\int_{G} \frac{|f(y)|}{\varphi(|x-y|)^{n-1}} d y\right\|_{L^{H}(G)} \leq C
$$


for every $f$ whenever $\|f\|_{L^{p}(G)} \leq 1$ and $1<p<\infty$. The boundedness of the Luxemburg norm follows by applying this inequality to $f /\|f\|_{L^{p}(G)}$ whenever $1<p<\infty$. Arguments in the case $p=1$ are similar.

We state the boundedness of the Luxemburg norm in the following corollary.

3.10. Corollary. Let $H$ be an Orlicz function and $\varphi$ be an increasing function as in Theorem 3.5. Let $G$ be an open set in $\mathbb{R}^{n}$.

If $1<p<n$, then there exists a constant $C$ such that the inequality

$$
\left\|\int_{G} \frac{|f(y)|}{\varphi(|\cdot-y|)^{n-1}} d y\right\|_{L^{H}(G)} \leq C\|f\|_{L^{p}(G)}
$$

holds for every $f \in L^{p}(G)$. Here the constant $C$ depends on $n, p, C_{\varphi}$, $C_{H}$, and the $\Delta_{2}$-constant of $H$ only.

If $p=1$, then there exists a constant $C_{1}$ such that the inequality

$$
\left\|\int_{G} \frac{|f(y)|}{\varphi(|\cdot-y|)^{n-1}} d y\right\|_{L^{H}(G)} \leq C_{1}\|f\|_{L \log L(G)}
$$

holds for every $f \in L \log L(G)$. Here the constant $C_{1}$ depends on $n, C_{\varphi}$, $C_{H}$, and the $\Delta_{2}$-constant of $H$ only.

3.11. Remark. (a) Theorem 3.5 reduces to the classical pointwise estimate for the Riesz potential $I_{\alpha} f$,

$$
I_{\alpha} f(x)=\int_{\mathbb{R}^{n}} \frac{|f(y)|}{|x-y|^{n-\alpha}} d y,
$$

that is, there exists a constant $C$ such that

$$
\left|I_{\alpha} f(x)\right|^{n p /(n-\alpha p)} \leq C M f(x)^{p}\|f\|_{L^{p}\left(\mathbb{R}^{n}\right)}^{\alpha p n p / n(n-\alpha p)},
$$

when $\alpha \in(0,1]$ and $1<p<n,[13,(3)$ in the proof of Theorem 1]. Indeed, if $f \in L^{p}\left(\mathbb{R}^{n}\right)$ is given and we choose $\varphi(t)=t^{\frac{n-\alpha}{n-1}}, h(t)=t^{\alpha}, \delta(t)=$ $\frac{t^{-\frac{p}{n}}}{\|f\|_{L^{p}\left(\mathbb{R}^{n}\right)}}$, and $H(t)=\frac{n-\alpha p}{n p} t^{n p /(n-\alpha p)}$, then the assumptions of Theorem 3.5 are valid. If $\alpha \in(1, n)$, then inequality (1.1) fails and we can not use the method of our proof for Theorem 3.5.

(b) The classical $(n p /(n-\alpha p), p)$-inequality for the Riesz potential $I_{\alpha} f$, that is, for $\alpha>0,1<p<\infty$, and $\alpha p<n$ there is a constant $C(n, p, \alpha)$ such that $\left\|I_{\alpha} f\right\|_{L^{n p /(n-\alpha p)}\left(\mathbb{R}^{n}\right)} \leq C(n, p, \alpha)\|f\|_{L^{p}\left(\mathbb{R}^{n}\right)}$ whenever $f \in$ $L^{p}\left(\mathbb{R}^{n}\right)$, [13, Theorem 1], is a special case of Theorem 3.8 with $\varphi(t)=t^{\frac{n-\alpha}{n-1}}$ and $H(t)=\frac{n-\alpha p}{n p} t^{n p /(n-\alpha p)}$ whenever $\alpha \in(0,1]$ and $1<p<n$.

(c) Trudinger's inequality [28, p. 479], [19, Theorem 1], [26, Theorem], and [13, Theorem 2] for functions with compact support follows from Theorem 3.8 when $\varphi(t)=t$ as in [13, p. 507].

More generally boundedness results to the Riesz operator $I_{\alpha}$ from an Orlicz space to another Orlicz space are found in [21], [27], [15], and [2]. Cianchi characterized the Orlicz functions which give the corresponding norm inequalities, [3, Theorem 2 (ii)]. Cianchi and Stroffolini gave 
simplified proofs, [4, Theorem 1, Corollary 1]. For recent developments we refer to [20].

\section{Pointwise estimates For FUnCtions Defined on IRREGULAR DOMAINS}

We are going to give new embedding results for $L_{p}^{1}$-functions, which are defined on domains with fractal boundaries. We recall the definition of very irregular John domains and give an integral representation to functions defined on these domains.

4.1. Definition. Let $\varphi:[0, \infty) \rightarrow[0, \infty)$ be a continuous, strictly increasing function. A bounded domain $D$ in $\mathbb{R}^{n}, n \geq 2$, is a $\varphi$-John domain if there exist a constant $c_{J}>0$ and a point $x_{0} \in D$ such that each point $x \in D$ can be joined to $x_{0}$ by a rectifiable curve $\gamma:[0, l] \rightarrow D$, parametrized by its arc length, such that $\gamma(0)=x, \gamma(l)=x_{0}, l \leq c_{J}$, and

$$
\varphi(t) \leq c_{J} \operatorname{dist}(\gamma(t), \partial D) \text { for all } t \in\left[0, c_{J}\right] .
$$

The point $x_{0}$ is called a John center of $D$ and the constant $c_{J}$ is called a John constant of $D$.

If a domain is a $\varphi$-John domain with a John center $x_{0}$, then it is a $\varphi$-John domain with any other $x \in D$, but the John constant might be different.

Lipschitz domains, classical John domains, and the so called $s$-John domains are examples of these domains. But there are more irregular domains such as the mushrooms domain studied in [10, 6. Example] and in [11, 6. Example].

The following lemma is needed to prove a pointwise integral representation to $L_{1}^{1}$-functions defined on a $\varphi$-John domain. Lemma 4.3 is a generalization of [9, Theorem 9.3] where the classical John domain, corresponding to the case $\varphi(t)=t$, is considered. For the function $\varphi(t)=t / \log \left(e+t^{-1}\right)$ the corresponding result has been proved in [11, Lemma 3.5]. The following inequality (4.2) is needed: There exists a constant $C_{\varphi}^{\prime}$ depending on $\varphi$ and $c_{J}$ only such that

$$
\varphi(t) \leq C_{\varphi}^{\prime} t \text { for all } t \in\left[0, c_{J}\right] .
$$

Namely, for a given John domain with a John constant $c_{J}$ by inequality (1.1) there exists a constant $C_{\varphi}$ such that

$$
\varphi(t) \leq C_{\varphi} \frac{\varphi\left(c_{J}\right)}{c_{J}} t=: C_{\varphi}^{\prime} t \quad \text { for all } t \in\left[0, c_{J}\right]
$$

4.3. Lemma. Let $\varphi:[0, \infty) \rightarrow[0, \infty)$ be a continuous, strictly increasing $\Delta_{2}$-function satisfying inequality (4.2). Let $D$ in $\mathbb{R}^{n}, n \geq 2$, be a $\varphi$-John domain with a John constant $c_{J}$ and a John center $x_{0} \in D$. Then for every $x \in D \backslash B\left(x_{0}\right.$, $\left.\operatorname{dist}\left(x_{0}, \partial D\right)\right)$ there exists a sequence of balls $\left(B\left(x_{i}, r_{i}\right)\right)$ such that $B\left(x_{i}, 2 r_{i}\right)$ is in $D, i=0,1, \ldots$, and for some constants $K=K\left(c_{J}, C_{\varphi}^{\prime}\right), N=N(n)$, and $M=M(n)$ 
(1) $B_{0}=B\left(x_{0}, \frac{1}{2} \operatorname{dist}\left(x_{0}, \partial D\right)\right)$;

(2) $\varphi\left(\operatorname{dist}\left(x, B_{i}\right)\right) \leq K r_{i}$, and $r_{i} \rightarrow 0$ as $i \rightarrow \infty$;

(3) no point of the domain $D$ belongs to more than $N$ balls $B\left(x_{i}, r_{i}\right)$; and

(4) $\left|B\left(x_{i}, r_{i}\right) \cup B\left(x_{i+1}, r_{i+1}\right)\right| \leq M\left|B\left(x_{i}, r_{i}\right) \cap B\left(x_{i+1}, r_{i+1}\right)\right|$.

Proof. The definition of $\varphi$ affects only to the property (2). Thus the proofs for (3) and (4) are the same as in [11, Lemma 3.5].

Let $x \in D \backslash B\left(x_{0}, \operatorname{dist}\left(x_{0}, \partial D\right)\right)$. Let $\gamma$ be a John curve joining $x$ to $x_{0}$, its arc length written as $l$. We write $B_{0}^{\prime}=B\left(x_{0}, \frac{1}{4} \operatorname{dist}\left(x_{0}, \partial D\right)\right)$ and consider the balls $B_{0}^{\prime}$ and

$$
B\left(\gamma(t), \frac{1}{4} \operatorname{dist}(\gamma(t), \partial D \cup\{x\})\right),
$$

where $t \in(0, l)$. By the Besicovitch covering theorem, there is a sequence of closed balls $\overline{B_{1}^{\prime}}, \overline{B_{2}^{\prime}}, \ldots$ and $\overline{B_{0}^{\prime}}$ that cover the set $\{\gamma(t)$ : $t \in[0, l]\} \backslash\{x\}$ and have a uniformly bounded overlap depending on $n$ only. We write $B\left(x_{i}, r_{i}\right)=2 B_{i}^{\prime}$ for every $i=0,1,2, \ldots$, where $x_{i}=\gamma\left(t_{i}\right)$, $t_{i} \in(0, l)$, and $r_{i}=\frac{1}{2} \operatorname{dist}\left(x_{i}, \partial D \cup\{x\}\right)$.

By the fact that $\varphi$ is an increasing function and by the definition of $\varphi$-John domain we obtain

$$
\varphi\left(\operatorname{dist}\left(x, B_{0}\right)\right) \leq \varphi(l) \leq c_{J} \operatorname{dist}\left(x_{0}, \partial D\right)=2 c_{J} r_{0} .
$$

Let us suppose then that $i \geq 1$. If $r_{i}=\frac{1}{2} \operatorname{dist}\left(x_{i}, x\right)$, then by inequality (4.2) we obtain

$$
\varphi\left(\operatorname{dist}\left(x, B\left(x_{i}, r_{i}\right)\right)\right) \leq C_{\varphi}^{\prime} \operatorname{dist}\left(x, B\left(x_{i}, r_{i}\right)\right) \leq 2 C_{\varphi}^{\prime} r_{i} .
$$

If $r_{i}=\frac{1}{2} \operatorname{dist}\left(x_{i}, \partial D\right)$, then the fact that $\varphi$ is increasing and the definition of a $\varphi$-John domain give

$$
\varphi\left(\operatorname{dist}\left(x, B\left(x_{i}, r_{i}\right)\right)\right) \leq \varphi\left(\operatorname{dist}\left(x, x_{i}\right)\right) \leq \varphi\left(t_{i}\right) \leq c_{J} \operatorname{dist}\left(x_{i}, \partial D\right)=2 c_{J} r_{i} .
$$

Thus, property (2) holds.

The following pointwise integral representation for $L_{1}^{1}$-functions defined on the classical John domain is well known, [24], [7]. The corresponding integral representation when $\varphi(t)=t / \log \left(e+t^{-1}\right)$ is proved in [11, Theorem 3.4]. For the sake of completeness we give the proof for the general function $\varphi$ here. Lemma 4.3 is essential to this proof.

4.4. Theorem. Let $\varphi:[0, \infty) \rightarrow[0, \infty)$ be a continuous, strictly increasing $\Delta_{2}$-function satisfying (4.2). Let $D$ in $\mathbb{R}^{n}, n \geq 2$, be a $\varphi$-John domain with a John constant $c_{J}$ and a John center $x_{0}$. Then there exists a finite constant $C$ such that for every $u \in L_{1}^{1}(D)$ and for almost every $x \in D$ the inequality

$$
\left|u(x)-u_{B\left(x_{0}, \operatorname{dist}\left(x_{0}, \partial D\right)\right)}\right| \leq C \int_{D} \frac{|\nabla u(y)|}{\varphi(|x-y|)^{n-1}} d y
$$

holds. 
Proof. If $x \in B\left(x_{0}, \operatorname{dist}\left(x_{0}, \partial D\right)\right)$, then

$\left|u(x)-u_{B\left(x_{0}, \operatorname{dist}\left(x_{0}, \partial D\right)\right)}\right| \leq \frac{\operatorname{diam}\left(B\left(x_{0}, \operatorname{dist}\left(x_{0}, \partial D\right)\right)\right)^{n}}{n\left|B\left(x_{0}, \operatorname{dist}\left(x_{0}, \partial D\right)\right)\right|} \int_{B\left(x_{0}, \operatorname{dist}\left(x_{0}, \partial D\right)\right)} \frac{|\nabla u(y)|}{|x-y|^{n-1}} d y$

by [5, Lemma 7.16]. Since by inequality (4.2) there is a constant $C_{\varphi}^{\prime}$ such that $\varphi(|x-y|)^{n-1} \leq\left(C_{\varphi}^{\prime}|x-y|\right)^{n-1}$, the claim follows for points $x \in B\left(x_{0}, \operatorname{dist}\left(x_{0}, \partial D\right)\right)$.

Let us then assume that $x \in D \backslash B\left(x_{0}, \operatorname{dist}\left(x_{0}, \partial D\right)\right)$. Let $\left(B_{i}\right)_{i=0}^{\infty}$ be a sequence of balls constructed in Lemma 4.3. Property (2) in Lemma 4.3 gives that $r_{i} \rightarrow 0$ and $\operatorname{dist}\left(x, B_{i}\right) \rightarrow 0$ whenever $i \rightarrow \infty$, since $\lim _{t \rightarrow 0+} \varphi(t)=0$ and $\varphi$ is continuously strictly increasing. Note that $\lim _{t \rightarrow 0+} \varphi(t)=0$ follows from the definition of $\varphi$-John domain by considering points near the boundary. Since $r_{i}=\frac{1}{2} \operatorname{dist}\left(x_{i}, \partial D \cup\{x\}\right)$, we obtain that $r_{i}=\frac{1}{2} \operatorname{dist}\left(x_{i}, x\right)$ when $i$ is large enough. Thus the Lebesgue differentiation theorem [25, Section 1, Corollary 1] imply that $u_{B_{i}} \rightarrow u(x)$ when $i \rightarrow \infty$ for almost every $x$. We obtain

$$
\begin{aligned}
\left|u(x)-u_{B_{0}}\right| & \leq \sum_{i=0}^{\infty}\left|u_{B_{i}}-u_{B_{i+1}}\right| \leq \sum_{i=0}^{\infty}\left(\left|u_{B_{i}}-u_{B_{i} \cap B_{i+1}}\right|+\left|u_{B_{i+1}}-u_{B_{i} \cap B_{i+1}}\right|\right) \\
& \leq \sum_{i=0}^{\infty}\left(\int_{B_{i} \cap B_{i+1}}\left|u(y)-u_{B_{i}}\right| d y+\int_{B_{i} \cap B_{i+1}}\left|u(y)-u_{B_{i+1}}\right| d y\right) .
\end{aligned}
$$

By property (4) in Lemma $4.3\left|u(x)-u_{B_{0}}\right| \leq 2 C \sum_{i=0}^{\infty}\left|B_{i}\right|^{-1} \int_{B_{i}}\left|u(y)-u_{B_{i}}\right| d y$. By using the $(1,1)$-Poincaré inequality in a ball $B_{i}$, [5, Section 7.8$]$, we obtain

$$
\left|u(x)-u_{B_{0}}\right| \leq C \sum_{i=0}^{\infty} r_{i} f_{B_{i}}|\nabla u(y)| d y .
$$

By (4.2) we have $\varphi\left(2 r_{i}\right) \leq 2 C_{\varphi}^{\prime} r_{i}$. Since $\varphi$ is strictly increasing, there exists the strictly increasing inverse function $\varphi^{-1}$ such that the inequality $\varphi^{-1}\left(2 C_{\varphi}^{\prime} r_{i}\right) \geq 2 r_{i}$ holds. Thus, for each $z \in B_{i}$ we obtain by property (2) in Lemma 4.3 that

$$
|x-z| \leq \operatorname{dist}\left(x, B_{i}\right)+2 r_{i} \leq \varphi^{-1}\left(K r_{i}\right)+2 r_{i} \leq 2 \varphi^{-1}\left(C r_{i}\right),
$$

$C=\max \left\{K, 2 C_{\varphi}^{\prime}\right\}$. Hence, we have $\varphi\left(\frac{1}{2}|x-z|\right) \leq C r_{i}$. By using this estimate and property (3) in Lemma 4.3 we obtain that

$$
\begin{aligned}
\left|u(x)-u_{B_{0}}\right| & \leq C \sum_{i=0}^{\infty} r_{i} \int_{B_{i}}|\nabla u(y)| d y \leq C \sum_{i=0}^{\infty} \int_{B_{i}} \frac{|\nabla u(y)|}{r_{i}^{n-1}} d y \\
& \leq C \sum_{i=0}^{\infty} \int_{B_{i}} \frac{|\nabla u(y)|}{\varphi\left(\frac{1}{2}|x-y|\right)^{n-1}} d y \leq C \int_{D} \frac{|\nabla u(y)|}{\varphi\left(\frac{1}{2}|x-y|\right)^{n-1}} d y .
\end{aligned}
$$

Since the function $\varphi$ safisfy the $\Delta_{2}$-condition, the claim follows. 


\section{ORLICZ EMBBEDING THEOREMS}

Continuous embeddings into Orlicz spaces of exponential type for domains with a cone condition are well known, [28, Theorem 1 , Theorem 2]; we also refer to [29], [23], [22]. We recall that Cianchi has proved sharp results for Orlicz-Sobolev spaces whenever relative isoperimetric inequalities are valid in the underlying domain, [2, Theorem 2 and Example 1]. His work covers Orlicz spaces of exponential type and more. In particular, classical John domains, that is, $\varphi(t)=t$, satisfy the Trudinger inequality, [2, Example1].

We formulate the new embedding results for $L_{p}^{1}$-functions defined on $\varphi$-John domains.

5.1. Theorem. Let $\varphi:[0, \infty) \rightarrow[0, \infty)$ be a continuous, strictly increasing $\Delta_{2}$ - function which satisfies condition (1.1). Let $H$ be an Orlicz function defined as in Theorem 3.5. Let $1<p<n$. If $D$ in $\mathbb{R}^{n}, n \geq 2$, is a $\varphi$-John domain with a John center $x_{0}$, then there exists a constant $C<\infty$ such that the inequality

$$
\int_{D} H\left(\mid u(x)-u_{B\left(x_{0}, \operatorname{dist}\left(x_{0}, \partial D\right) \mid\right)} d x \leq C\right.
$$

holds whenever $u \in L_{p}^{1}(D)$ and $\|\nabla u\|_{L^{p}(D)} \leq 1$; the constant $C$ does not depend on the function $u$.

If $p=1$, we need an extra assumption on the Orlicz function $H$.

5.2. Theorem. Let $\varphi:[0, \infty) \rightarrow[0, \infty)$ be a continuous, strictly increasing $\Delta_{2}$-function which satisfies condition (1.1). Let $H$ be an Orlicz function defined as in Theorem 3.5. Let $D$ in $\mathbb{R}^{n}, n \geq 2$, be a $\varphi$-John domain. If

$$
\sum_{j=1}^{\infty} H\left(2^{-j}\right)<\infty,
$$

then there exists a finite constant $C$ such that the inequality

$$
\int_{D} H\left(\left|u(x)-u_{B\left(x_{0}, \operatorname{dist}\left(x_{0}, \partial D\right)\right)}\right|\right) d x \leq C
$$

holds for every $u \in L_{1}^{1}(D)$ when $\|\nabla u\|_{L^{1}(D)} \leq 1$; the constant $C$ does not depend on the function $u$.

We state the corresponding norm inequalities next.

5.4. Corollary. If $1<p<n$, let $\varphi, H$, and $D$ be defined as in Theorem 5.1. If $p=1$, let $\varphi, H$, and $D$ be defined as in Theorem 5.2. Then there exists a constant $C<\infty$ such that the inequality

$$
\left\|u-u_{D}\right\|_{L^{H}(D)} \leq C\|\nabla u\|_{L^{p}(D)}
$$

holds for every $u \in L_{p}^{1}(D)$; the constant $C$ does not depend on the function $u$. 
The pointwise estimate in Theorem 4.4 is crucial for the proofs.

Proof of the embedding result Theorem 5.1. Let $u \in L_{p}^{1}(D)$. Then, by $[17,1.1 .2$, Theorem $] u \in L_{\text {loc }}^{p}(D)$. Let $x_{0} \in D$ be a John center of $D$. Theorem 4.4 and Theorem 3.8 imply the claim.

The proof of the embedding result is more tedious when $p=1$.

Proof of the embedding result Theorem 5.2. Let us consider functions $u \in L_{1}^{1}(D)$ such that $\|\nabla u\|_{L^{1}(D)} \leq 1$. The center ball $B\left(x_{0}, \operatorname{dist}\left(x_{0}, \partial D\right)\right)$ is written as $B$. We show that there exists a constant $C<\infty$ such that the inequality

$$
\int_{D} H\left(\left|u(x)-u_{B}\right|\right) d x \leq C
$$

holds whenever $\|\nabla u\|_{L^{1}(D)} \leq 1$. First we estimate

$$
\int_{D} H\left(\left|u(x)-u_{B}\right|\right) d x \leq \sum_{j \in \mathbb{Z}} \int_{\left\{x \in D: 2^{j}<\left|u(x)-u_{B}\right| \leq 2^{j+1}\right\}} H\left(2^{j+1}\right) d x .
$$

Let us define $v_{j}(x)=\max \left\{0, \min \left\{\left|u(x)-u_{B}\right|-2^{j}, 2^{j}\right\}\right\}$ for all $x \in D$. If $x \in\left\{x \in D: 2^{j}<\left|u(x)-u_{B}\right| \leq 2^{j+1}\right\}$, then $v_{j-1}(x) \geq 2^{j-1}$. We obtain

$$
\int_{D} H\left(\left|u(x)-u_{B}\right|\right) d x \leq \sum_{j \in \mathbb{Z}} \int_{\left\{x \in D: v_{j}(x) \geq 2^{j}\right\}} H\left(2^{j+2}\right) d x .
$$

By the triangle inequality and Theorem 4.4 we have

$$
v_{j}(x) \leq\left|v_{j}(x)-\left(v_{j}\right)_{B}\right|+\left|\left(v_{j}\right)_{B}\right| \leq C \int_{D} \frac{\left|\nabla v_{j}(y)\right|}{\varphi(|x-y|)^{n-1}} d y+\left|\left(v_{j}\right)_{B}\right|
$$

for almost every $x \in D$. By the $(1,1)$-Poincaré inequality in a ball $B$, [5, Section 7.8], there exists a constant $C$ such that

$$
\left|\left(v_{j}\right)_{B}\right|=\left(v_{j}\right)_{B}=f_{B} v_{j}(x) d x \leq f_{B}\left|u(x)-u_{B}\right| d x \leq C f_{B}|\nabla u(x)| d x \leq C|B|^{-1} .
$$

Thus, by the definition of $B$ the number $\left|\left(v_{j}\right)_{B}\right|$ is bounded by a constant depending on $n$ and the distance between the John center and the boundary of $D$ only. We write

$$
I_{\varphi}\left(\nabla v_{j}\right)(x)=\int_{D} \frac{\left|\nabla v_{j}(y)\right|}{\varphi(|x-y|)^{n-1}} d y .
$$

We continue to estimate the right hand side of inequality (5.6)

$$
\begin{aligned}
& \int_{D} H\left(\left|u(x)-u_{B}\right|\right) d x \leq \sum_{j \in \mathbb{Z}} \int_{\left\{x \in D: C I_{\varphi}\left(\nabla v_{j}\right)(x)+C \geq 2^{j}\right\}} H\left(2^{j+2}\right) d x \\
& \quad \leq \sum_{j \in \mathbb{Z}} \int_{\left\{x \in D: C I_{\varphi}\left(\nabla v_{j}\right)(x) \geq 2^{j-1}\right\}} H\left(2^{j+2}\right) d x+\sum_{j=-\infty}^{j_{0}} \int_{D} H\left(2^{j+2}\right) d x .
\end{aligned}
$$


By (5.3) we obtain

$$
\sum_{j=-\infty}^{j_{0}} \int_{D} H\left(2^{j+2}\right) d x=|D| \sum_{j=-\infty}^{j_{0}} H\left(2^{j+2}\right) \leq C|D| .
$$

Then, we will find an upper bound for the sum

$$
\sum_{j \in \mathbb{Z}} \int_{\left\{x \in D: C I_{\varphi}\left(\nabla v_{j}\right)(x) \geq 2^{j-1}\right\}} H\left(2^{j+2}\right) d x .
$$

Since $\left\|\nabla v_{j}\right\|_{L^{1}(D)} \leq\|\nabla u\|_{L^{1}(D)} \leq 1$, Theorem 3.5 implies that

$$
\begin{aligned}
\sum_{j \in \mathbb{Z}} \int_{\left\{x \in D: C I_{\varphi}\left(\nabla v_{j}\right)(x) \geq 2^{j-1}\right\}} H\left(2^{j+2}\right) d x & \leq \sum_{j \in \mathbb{Z}} \int_{\left\{x \in D: H\left(C I_{\varphi}\left(\nabla v_{j}\right)(x)\right) \geq H\left(2^{j-1}\right)\right\}} H\left(2^{j+2}\right) d x \\
& \leq \sum_{j \in \mathbb{Z}} \int_{\left\{x \in D: C M\left|\nabla v_{j}\right|(x) \geq H\left(2^{j-1}\right)\right\}} H\left(2^{j+2}\right) d x .
\end{aligned}
$$

We choose for every $x \in\left\{x \in D: C M\left|\nabla v_{j}\right|(x) \geq H\left(2^{j-2}\right)\right\}$ a ball $B\left(x, r_{x}\right)$, centered at $x$ and with radius $r_{x}$ depending on $x$, such that

$$
C f_{B\left(x, r_{x}\right)}\left|\nabla v_{j}(y)\right| d y \geq \frac{1}{2} H\left(2^{j-1}\right)
$$

with the understanding that $\left|\nabla v_{j}\right|$ is zero outside $D$. By the Besicovitch covering theorem (or the 5-covering theorem) we obtain a subcovering $\left\{B_{k}\right\}_{k=1}^{\infty}$ so that we may estimate by the $\Delta_{2}$-condition of $H$

$$
\begin{aligned}
& \sum_{j \in \mathbb{Z}} \int_{\left\{x \in D: C I_{\varphi}\left(\nabla v_{j}\right)(x) \geq 2^{j-1}\right\}} H\left(2^{j+2}\right) d x \leq \sum_{j \in \mathbb{Z}} \sum_{k=1}^{\infty} \int_{B_{k}} H\left(2^{j+2}\right) d x \\
& \quad \leq \sum_{j \in \mathbb{Z}} \sum_{k=1}^{\infty}\left|B_{k}\right| H\left(2^{j+2}\right) \leq \sum_{j \in \mathbb{Z}} \sum_{k=1}^{\infty} C\left|B_{k}\right| \frac{H\left(2^{j+2}\right)}{H\left(2^{j-1}\right)} \int_{B_{k}}\left|\nabla v_{j}(y)\right| d y \\
& \quad \leq C \sum_{j \in \mathbb{Z}} \int_{D}\left|\nabla v_{j}(y)\right| d y .
\end{aligned}
$$

Let $E_{j}=\left\{x \in D: 2^{j}<\left|u(x)-u_{B}\right| \leq 2^{j+1}\right\}$. Since $\left|\nabla v_{j}\right|$ is zero almost everywhere in $D \backslash E_{j}$ and $|\nabla u(x)|=\sum_{j}\left|\nabla v_{j}(x)\right| \chi_{E_{j}}(x)$ for almost every $x \in D$, we obtain

$$
\sum_{j \in \mathbb{Z}} \int_{\left\{x \in D: C I_{\varphi}\left(\nabla v_{j}\right)(x) \geq 2^{j-1}\right\}} H\left(2^{j+2}\right) d x \leq C \int_{D}|\nabla u(y)| d y \leq C .
$$

Estimates (5.7), (5.8), and (5.9) imply inequality (5.5).

Proof of Corollary 5.4. Let us write $B=B\left(x_{0}, \operatorname{dist}\left(x_{0}, \partial D\right)\right)$. Theorem 5.1 for $1<p<n$ and Theorem 5.2 for $p=1$ yield $\left\|u-u_{B}\right\|_{L^{H}(D)} \leq C$ for every $u \in L_{p}^{1}(D)$ with $\|\nabla u\|_{L^{p}(D)} \leq 1$. By using this inequality for $u /\|\nabla u\|_{L^{p}(D)}$ we obtain

$$
\left\|u-u_{B}\right\|_{L^{H}(D)} \leq C\|\nabla u\|_{L^{p}(D)} .
$$


By the triangle inequality $\left\|u-u_{D}\right\|_{L^{H}(D)} \leq\left\|u-u_{B}\right\|_{L^{H}(D)}+\left\|u_{B}-u_{D}\right\|_{L^{H}(D)}$ and furthermore for some constant $C$

$$
\begin{aligned}
\left\|u_{B}-u_{D}\right\|_{L^{H}(D)} & =\left|u_{B}-u_{D}\right|\|1\|_{L^{H}(D)} \leq\|1\|_{L^{H}(D)}\left\|u-u_{B}\right\|_{L^{1}(D)} \\
& \leq C\|1\|_{L^{H}(D)}\left\|u-u_{B}\right\|_{L^{H}(D)} .
\end{aligned}
$$

As an application of Theorems 5.1 and 5.2 we obtain the following corollary. By Remark 3.4 we may replace the assumption $p<n$ by the assumption $p<n /(n-\alpha(n-1))$.

5.10. Corollary (Theorem 4.1 in [10]). Let $1 \leq p<n /(n-\alpha(n-1)$ ) be given. Let $\alpha \in[1,1+1 /(n-1))$ and $\beta \geq 0$. Let $\varphi:(0, \infty) \rightarrow \mathbb{R}$ and $H:[0, \infty) \rightarrow \mathbb{R}$ be the functions

$$
\varphi(t)=\frac{t^{\alpha}}{\log ^{\beta}\left(e+t^{-1}\right)} \quad \text { and } \quad H(t)=\left(\frac{t}{\log ^{\beta(n-1)}(m+t)}\right)^{\frac{n p}{\alpha p(n-1)+n(1-p)}}
$$

where $m=m(n, p) \geq e$. If $D$ in $\mathbb{R}^{n}, n \geq 2$, is a $\varphi$-John domain, then there is a constant $C$ such that the inequality

$$
\int_{D} H\left(\left|u(x)-u_{B\left(x_{0}, \operatorname{dist}\left(x_{0}, \partial D\right)\right)}\right|\right) d x \leq C
$$

holds for every $u \in L_{p}^{1}(D)$ when $\|\nabla u\|_{L^{p}(D)} \leq 1$. The constant $C$ does not depend on the function $u$.

Corollary 5.10 recovers the well known case when $\alpha=1$ and $\beta=0$. Corollary 5.10 with $\alpha>1, \beta=0$ and $p=1$ is sharp but, with $\alpha>1$, $\beta=0$ and $p>1$ it is not sharp. Namely, the exponent in the Orlicz function $H$ should be $\frac{n p}{\alpha(n-1)-p+1}$, and not $\frac{n p}{\alpha p(n-1)+n(1-p)}$, according to $[8$, p. 437] and [14, Theorem 2.3]. We propose a conjecture that $\frac{n p}{\alpha(n-1)-p+1}$ is the right exponent in the case $\beta>0$ also. We note that our method based on the modified Riesz potential does not give a better exponent than $\frac{n p}{\alpha p(n-1)+n(1-p)}$, see Theorem 6.5.

We give a detailed proof Corollary 5.10, since the proof shows why the values of $\alpha$ should have the upper bound $n /(n-1)$. In Remark 5.11 we will point out that the upper bound $n /(n-1)$ is the best possible with this Hedberg-type method for the modified Riesz potentials.

Proof of Corollary 5.10. Let $\alpha \in[1,1+1 /(n-1))$ and $\beta \geq 0$. When $\varphi(t)=\frac{t^{\alpha}}{\log ^{\beta}\left(e+t^{-1}\right)}$, calculations show that the $\Delta_{2}$-condition of $\varphi$ and inequality (1.1) hold.

In order to have condition (3.2) we substitute $\varphi$ to the left hand side of (3.2) and estimate, for $\alpha<\frac{n}{n-1}$,

$$
\begin{aligned}
\sum_{k=1}^{\infty} \frac{\left(2^{-k} t\right)^{n}}{\varphi\left(2^{-k} t\right)^{n-1}} & \leq t^{n-\alpha(n-1)} \sum_{k=1}^{\infty} 2^{-k(n-\alpha(n-1)} \log ^{\beta(n-1)}\left(2^{k}\left(e+\frac{1}{t}\right)\right) \\
& \leq C t^{n-\alpha(n-1)} \log ^{\beta(n-1)}\left(e+\frac{1}{t}\right) .
\end{aligned}
$$


Thus, we may choose

$h(t)=C t^{n+(1-n) \alpha} \log ^{\beta(n-1)}\left(e+t^{-1}\right) \quad$ and $\quad H(t)=\left(\frac{t}{\log ^{\beta(n-1)}(m+t)}\right)^{\frac{n p}{\alpha p(n-1)+n-n p}}$.

Conditions $\Delta_{2}$ and (5.3) for the function $H$ hold clearly.

We choose $\delta:(0, \infty) \rightarrow(0, \infty), \delta(t)=t^{-\frac{p}{n}}$, and show that condition (3.6) holds with $\delta, h$, and $H$. By substituting $h$ and $\delta$ to the left hand side of (3.6) we obtain

$$
H\left(h(\delta(t)) t+\varphi(\delta(t))^{1-n} \delta(t)^{n\left(1-\frac{1}{p}\right)}=H\left(2 C t^{\frac{\alpha p(n-1)+n-n p}{n}} \log ^{\beta(n-1)}\left(e+t^{\frac{p}{n}}\right)\right) .\right.
$$

The definition of $H$ and straightforward estimates imply

$$
\begin{aligned}
& H\left(h(\delta(t)) t+\varphi(\delta(t))^{1-n} \delta(t)^{n\left(1-\frac{1}{p}\right.}\right) \\
& \leq \frac{C t^{p}\left(\log ^{\beta(n-1)}\left(e+t^{\frac{p}{n}}\right)\right)^{\frac{p n}{\alpha p(n-1)+n-n p}}}{\left(\log ^{\beta(n-1)}\left(m+2 C t^{\frac{\alpha p(n-1)+n-n p}{n}} \log ^{\beta(n-1)}\left(e+t^{\frac{p}{n}}\right)\right)\right)^{\frac{p n}{\alpha_{p(n-1)+n-n p}}}} \\
& \quad \leq C t^{p}\left(\frac{\log ^{\beta(n-1)}\left(e+t^{\frac{p}{n}}\right)}{\log ^{\beta(n-1)}\left(m+2 C t^{\frac{\alpha p(n-1)+n-n p}{n}}\right)}\right)^{\frac{p n}{\alpha p(n-1)+n-n p}} \leq C t^{p} .
\end{aligned}
$$

Thus the claim follows by Theorems 5.1 and 5.2.

5.11. Remark. We emphasize that the assumption $\alpha<1+\frac{1}{n-1}$ is natural when we consider the function $\varphi(t)=\frac{t^{\alpha}}{\log ^{\beta}\left(e+t^{-1}\right)}$. Namely, if we assume that $\alpha \geq 1+\frac{1}{n-1}$ and $\beta \geq 0$ and choose $f \equiv 1$ in $D$, then we obtain that

$$
\begin{aligned}
& \int_{D} \frac{|f(y)|}{\varphi(|x-y|)^{n-1}} d y \geq \int_{B(x, \min \{1, \operatorname{dist}(x, \partial D)\})} \frac{\log ^{\beta(n-1)}\left(e+|x-y|^{-1}\right)}{|x-y|^{\alpha(n-1)}} d y \\
& \quad \geq \int_{B(x, \min \{1, \operatorname{dist}(x, \partial D)\})} \frac{1}{|x-y|^{\alpha(n-1)}} d y \geq \int_{B(x, \min \{1, \operatorname{dist}(x, \partial D)\})} \frac{1}{|x-y|^{n}} d y=\infty
\end{aligned}
$$

for every $x \in D$.

\section{Sharpness of the RESUlts}

In this section we study sharpness of the norm inequalities

$$
\left\|\int_{D} \frac{|u(z)|}{\varphi(|\cdot-z|)} d z\right\|_{L^{H}(D)} \leq C\|u\|_{L^{p}(D)}
$$

and $\left\|u-u_{D}\right\|_{L^{H}(D)} \leq C\|\nabla u\|_{L^{p}(D)}$. We start from the latter inequality.

Let $\varphi:[0, \infty) \rightarrow[0, \infty)$ be a continuous, strictly increasing $\Delta_{2^{-}}$ function which satisfies condition (1.1). We give a sufficient condition to the function $H$ in Theorem 6.2 so that the corresponding inequality in Theorem 5.1 fails. We do it by constructing a mushrooms-type domain. Mushrooms-type domains can be found in [18], [17], [12], [11], [10]. By using Theorem 6.2 we show that the embedding in Theorem 5.2 is sharp. 
Next we construct the mushrooms-type domain. Let $\left(r_{m}\right)$ be a decreasing sequence converging to zero. Let $Q_{m}, m=1,2, \ldots$, be a closed cube in $\mathbb{R}^{n}$ with side length $2 r_{m}$. Let $P_{m}, m=1,2, \ldots$, be a closed rectangle in $\mathbb{R}^{n}$ which has side length $r_{m}$ for one side and $2 \varphi\left(r_{m}\right)$ for the remaining $n-1$ sides. Let $Q_{0}=[0,1]^{n}$. We attach $Q_{m}$ and $P_{m}$ together creating 'mushrooms' which we then attach, as pairwise disjoint sets, to one side of $Q_{0}$. We have to assume here that $\varphi\left(r_{m}\right) \leq r_{m}$. We attach the mushrooms to the side that lies in the hyperplane $x_{2}=1$. We wish to define a domain that is symmetric with respect to the hyperplane $x_{2}=\frac{1}{2}$. Thus, let $Q_{m}^{*}$ and $P_{m}^{*}$ be the images of the sets $Q_{m}$ and $P_{m}$, respectively, under the reflection across the hyperplane $x_{2}=\frac{1}{2}$. We define

$$
G=\operatorname{int}\left(Q_{0} \cup \bigcup_{m=1}^{\infty}\left(Q_{m} \cup P_{m} \cup Q_{m}^{*} \cup P_{m}^{*}\right)\right) .
$$

We give a sufficient condition to the Orlicz function $H$ so that the corresponding Orlicz embedding result in Theorem 5.1 fails.

6.2. Theorem. Let $p \geq 1$. Let $\varphi:[0, \infty) \rightarrow[0, \infty)$ be a continuous, strictly increasing $\Delta_{2}$-function which satisfies condition (1.1). Suppose that there exists $t_{0}>0$ such that $\varphi(t) \leq t$ for $0<t<t_{0}$. Let $G$ in $\mathbb{R}^{n}$, $n \geq 2$, be a mushrooms-type domain constructed as in (6.1). If $H$ is an Orlicz function which satisfies the $\Delta_{2}$-property and the condition

$$
\lim _{t \rightarrow 0^{+}} t^{n} H\left(\left(\frac{t^{p-1}}{\varphi(t)^{n-1}}\right)^{1 / p}\right)=\infty,
$$

then there exists a sequence of functions $\left(u_{k}\right)$ in $L_{p}^{1}(G)$ such that $\left\|\nabla u_{k}\right\|_{L^{p}(G)}=$ 1 for every $k$ and

$$
\int_{D} H\left(\left|u_{k}(x)-\left(u_{k}\right)_{D}\right|\right) d x \rightarrow \infty \quad \text { as } \quad k \rightarrow \infty .
$$

Proof. Let us define a sequence of piecewise linear continuous functions $\left(u_{k}\right)_{k=1}^{\infty}$ by setting

$$
u_{k}(x):= \begin{cases}F\left(r_{k}\right) & \text { in } Q_{k} \\ -F\left(r_{k}\right) & \text { in } Q_{k}^{*} \\ 0 & \text { in } Q_{0},\end{cases}
$$

where the function $F$ will be given in (6.3). Then the integral average of $u_{k}$ over $G$ is zero for each $k$.

The gradient of $u_{k}$ differs from zero in $P_{m} \cup P_{m}^{*}$ only and

$$
\left|\nabla u_{k}(x)\right|=\frac{F\left(r_{m}\right)}{r_{m}} \text {, when } x \in P_{m} \cup P_{m}^{*} \text {. }
$$

Note that

$$
\int_{G}\left|\nabla u_{k}(x)\right|^{p} d x=2 \int_{P_{m}}\left(\frac{F\left(r_{m}\right)}{r_{m}}\right)^{p} d x=2 r_{m}\left(\varphi\left(r_{m}\right)\right)^{n-1} \frac{F\left(r_{m}\right)^{p}}{r_{m}^{p}} .
$$


We require that $\int_{G}\left|\nabla u_{k}(x)\right|^{p} d x=1$. Hence,

$$
F\left(r_{m}\right)=\left(\frac{r_{m}^{p-1}}{2 \varphi\left(r_{m}\right)^{n-1}}\right)^{1 / p} .
$$

Since $\left(u_{k}\right)_{G}=0$ and $u_{k}$ is a constant in $Q_{m}$ we have

$$
\int_{G} H\left(\left|u_{k}(x)-\left(u_{k}\right)_{G}\right|\right) d x \geq 2 \int_{Q_{m}} H\left(F\left(r_{m}\right)\right) d x=2 r_{m}^{n} H\left(F\left(r_{m}\right)\right) .
$$

Hence, by (6.3), the $\Delta_{2}$-condition, and the assumption we have

$$
\begin{aligned}
r_{m}^{n} H\left(F\left(r_{m}\right)\right) & =r_{m}^{n} H\left(\left(\frac{r_{m}{ }^{p-1}}{2 \varphi\left(r_{m}\right)^{n-1}}\right)^{1 / p}\right) \geq r_{m}^{n} H\left(\frac{1}{2}\left(\frac{r_{m}{ }^{p-1}}{\varphi\left(r_{m}\right)^{n-1}}\right)^{1 / p}\right) \\
& \geq \frac{1}{C_{H}^{\Delta_{2}}} r_{m}^{n} H\left(\left(\frac{r_{m}{ }^{p-1}}{\varphi\left(r_{m}\right)^{n-1}}\right)^{1 / p}\right) \rightarrow \infty, \text { whenever } \quad m \rightarrow \infty .
\end{aligned}
$$

Theorem 6.2 implies that condition (3.6) in Theorem 5.2, in the $p=1$ case, is sharp.

6.4. Remark. Let $H$ be an Orlicz $\Delta_{2}$-function which satisfies condition (5.3). Let us assume that (3.6) holds with $\delta(t)=t^{-\frac{p}{n}}$ in the case $p=1$, that is,

$$
H\left(h\left(t^{-\frac{1}{n}}\right) t+\varphi\left(t^{-\frac{1}{n}}\right)^{1-n}\right) \leq C_{H} t \text { for all } t \geq 0 .
$$

Then we obtain with every function $h$ that

$$
\begin{aligned}
\lim _{t \rightarrow 0^{+}} t^{n} H\left(\frac{1}{\varphi(t)^{n-1}}\right) & \leq \lim _{t \rightarrow 0^{+}} t^{n} H\left(h(t) t^{-n}+\varphi(t)^{1-n}\right) \\
& \leq \lim _{t \rightarrow \infty} t^{-1} H\left(h\left(t^{-1 / n}\right) t+\varphi\left(t^{-\frac{1}{n}}\right)^{1-n}\right) \leq C_{H} .
\end{aligned}
$$

Next we study the modified Riesz potential in $\mathbb{R}^{n}$.

6.5. Theorem. Let $\varepsilon, \delta, \beta \geq 0$ and let $\alpha \in[1,1+1 /(n-1))$. Let $1 \leq p<$ $n /(n-\alpha(n-1))$ be given. Let $\varphi:(0, \infty) \rightarrow \mathbb{R}$ and $H:[0, \infty) \rightarrow \mathbb{R}$ be the functions

$$
\varphi(t)=\frac{t^{\alpha}}{\log ^{\beta}\left(e+t^{-1}\right)} \quad \text { and } \quad H(t)=\left(\frac{t}{\log ^{\beta(n-1)-\delta}(m+t)}\right)^{\frac{n p}{\alpha p(n-1)+n(1-p)}+\varepsilon},
$$

where $m \geq e$. If $\varepsilon>0$ or $\delta>0$, then there exists a sequence of functions $\left(u_{k}\right)$ in $L^{p}\left(\mathbb{R}^{n}\right)$ such that $\left\|u_{k}\right\|_{L^{p}\left(\mathbb{R}^{n}\right)} \leq C$ and

$$
\int_{\mathbb{R}^{n}} H\left(\int_{\mathbb{R}^{n}} \frac{\left|u_{k}(z)\right|}{\varphi(|x-z|)^{n-1}} d z\right) d x \rightarrow \infty \quad \text { as } \quad k \rightarrow \infty .
$$

Proof. Let $A>0$. We will fix $f \in L^{p}\left(\mathbb{R}^{n}\right)$ later. By changing the variables we obtain

$$
\left\|A^{\frac{n}{p}} f(A x)\right\|_{L^{p}(G)}=\left(\int_{\mathbb{R}^{n}} A^{n}|f(A x)|^{p} d x\right)^{\frac{1}{p}}=\left(\int_{\mathbb{R}^{n}}|f(y)|^{p} d y\right)^{\frac{1}{p}}=\|f\|_{L^{p}\left(\mathbb{R}^{n}\right)} .
$$


On the other hand, by changing the variables, $A x=z$ and $A y=\omega$, we obtain

$$
\begin{aligned}
& \int_{\mathbb{R}^{n}} H\left(\int_{\mathbb{R}^{n}} \frac{\left|A^{\frac{n}{p}} f(A x)\right|}{\varphi(|x-y|)^{n-1}} d x\right) d y=\int_{\mathbb{R}^{n}} H\left(\int_{\mathbb{R}^{n}} \frac{A^{\frac{n}{p}}|f(z)|}{A^{n} \varphi\left(\left|\frac{z}{A}-y\right|\right)^{n-1}} d z\right) d y \\
& =\int_{\mathbb{R}^{n}} A^{-n} H\left(A^{\frac{n}{p}-n} \int_{\mathbb{R}^{n}} \frac{|f(z)|}{\varphi\left(A^{-1}|z-\omega|\right)^{n-1}} d z\right) d \omega .
\end{aligned}
$$

Thus, by Fatou's lemma

$$
\begin{aligned}
\lim _{A \rightarrow \infty} & \int_{\mathbb{R}^{n}} H\left(\int_{\mathbb{R}^{n}} \frac{\left|A^{\frac{n}{p}} f(A x)\right|}{\varphi(|x-y|)^{n-1}} d x\right) d y \\
& \geq \int_{\mathbb{R}^{n}} \lim _{A \rightarrow \infty} A^{-n} H\left(A^{\frac{n}{p}-n} \int_{\mathbb{R}^{n}} \frac{|f(z)|}{\varphi\left(A^{-1}|z-\omega|\right)^{n-1}} d z\right) d \omega .
\end{aligned}
$$

Let $f(x)=1$ when $x \in B(0,2)$ and let $f(x)=0$ otherwise. Hence, by substituting $\varphi(t)=\frac{t^{\alpha}}{\log ^{\beta}\left(e+t^{-1}\right)}$ we obtain that for every $\omega \in B(0,1)$

$$
\begin{gathered}
A^{-n} H\left(A^{\frac{n}{p}-n} \int_{\mathbb{R}^{n}} \frac{|f(z)|}{\varphi\left(A^{-1}|z-\omega|\right)^{n-1}} d z\right) \\
=A^{-n} H\left(A^{\frac{n}{p}-n} \int_{\mathbb{R}^{n}} \frac{|f(z)| \log ^{\beta(n-1)}(e+A /|z-\omega|)}{A^{-\alpha(n-1)}|z-\omega|^{\alpha(n-1)}} d z\right) \\
\quad \geq A^{-n} H\left(A^{\frac{n}{p}-n+\alpha(n-1)} \int_{B\left(\omega, \frac{1}{2}\right)} \frac{\log ^{\beta(n-1)}(e+A)}{|z-\omega|^{\alpha(n-1)}} d z\right) .
\end{gathered}
$$

Since $\int_{B\left(\omega, \frac{1}{2}\right)} \frac{1}{|z-\omega|^{\alpha(n-1)}} d z \geq C>0$ for every $\omega \in B(0,1)$ and $H$ is an increasing function, we may estimate

$$
\begin{aligned}
& \lim _{A \rightarrow \infty} A^{-n} H\left(A^{\frac{n}{p}-n} \int_{\mathbb{R}^{n}} \frac{|f(z)|}{\varphi\left(A^{-1}|z-\omega|\right)^{n-1}} d z\right) \\
& \quad \geq \lim _{A \rightarrow \infty} A^{-n} H\left(C A^{\frac{n}{p}-n+\alpha(n-1)} \log ^{\beta(n-1)}(e+A)\right) .
\end{aligned}
$$

By substituting $H$,

$$
H(t)=\left(\frac{t}{\log ^{\beta(n-1)-\delta}(m+t)}\right)^{\frac{n p}{\alpha p(n-1)+n(1-p)}+\varepsilon},
$$

we obtain

$$
\begin{aligned}
& \lim _{A \rightarrow \infty} A^{-n} H\left(A^{\frac{n}{p}-n} \int_{\mathbb{R}^{n}} \frac{|f(z)|}{\varphi\left(A^{-1}|z-\omega|\right)^{n-1}} d z\right) \\
& =\lim _{A \rightarrow \infty} C A^{\varepsilon\left(\frac{n}{p}-n+\alpha(n-1)\right)}\left(\frac{\log ^{\beta(n-1)}(e+A)}{\log ^{\beta(n-1)-\delta}\left(C A^{\frac{n}{p}-n+\alpha(n-1)} \log ^{\beta(n-1)}(e+A)\right)}\right)^{\frac{n p}{\alpha p(n-1)+n(1-p)}+\varepsilon} .
\end{aligned}
$$

Note that $1 \leq p<n /(n-\alpha(n-1))$ implies that $\frac{n}{p}-n+\alpha(n-1)>0$. If $\varepsilon>0$, then $A^{\varepsilon\left(\frac{n}{p}-n+\alpha(n-1)\right)} \rightarrow \infty$ as $A \rightarrow \infty$, and thus the last limit is infinite for every $\omega \in B(0,1)$. If $\delta>0$ (and $\beta \geq 0$ ), then the term in the 
brackets tends to infinity as $A \rightarrow \infty$, and thus the last limit is infinite for every $\omega \in B(0,1)$. Hence, in both cases we obtain

$$
\lim _{A \rightarrow \infty} \int_{\mathbb{R}^{n}} H\left(\int_{\mathbb{R}^{n}} \frac{\left|A^{\frac{n}{p}} f(A x)\right|}{\varphi(|x-y|)^{n-1}} d x\right) d y=\infty .
$$

6.6. Remark. By Theorem 6.5 the exponents $\frac{n p}{\alpha p(n-1)+n(1-p)}$ and $\beta(n-1)$ in Corollary 5.10 are the best possible in the sense that our method based on the use of the modified Riesz potential cannot give a better exponent.

\section{REFERENCES}

[1] R. A. Adams and J. J. F. Fournier, Sobolev Spaces Second edition, Pure and Applied Mathematics Series, 140. Elsevier/Academic Press, Amsterdam, 2003.

[2] A. Cianchi, A sharp embedding theorem for Orlicz-Sobolev spaces, Indiana Univ. Math. J. 45 (1996), 39-65.

[3] A. Cianchi, Strong and weak type inequalities for some classical operators in Orlicz spaces, J. London Math. Soc. (2) 60 (1999), 187-202.

[4] A. Cianchi and B. Stroffolini, An extension of Hedberg's convolution inequality and applications, J. Math. Anal. Appl. 227 (1998), 166-186.

[5] D. Gilbarg and N. S. Trudinger, Elliptic Partial Differential Equations of Second Order. Reprint of the 1998 edition. Classics in Mathematics. SpringerVerlag, Berlin, 2001.

[6] D. J. H. Garling, Inequalities, A Journey into Linear Analysis. Cambridge University Press, Cambridge, 2007.

[7] P. Hajłasz, Sobolev inequalities, truncation method, and John domains, Papers on Analysis: A volume dedicated to Olli Martio on the occasion of his 60th birthday. Edited by J. Heinonen, T. Kilpeläinen, and P. Koskela, Report Univ. Jyväskylä, 83, University of Jyväskylä, Jyväskylä, 2001, pp. 109-126.

[8] P. Hajłasz and P. Koskela, Isoperimetric inequalities and imbedding theorems in irregular domains, J. London Math. Soc. (2)58 (1998), no. 2, 425-450.

[9] P. Hajłasz and P. Koskela, Sobolev met Poincaré, Mem. Amer. Math. Soc. 145 (2000), no. 688, x+101 pp.

[10] P. Harjulehto and R. Hurri-Syrjänen, An embedding into an Orlicz space for $L_{1}^{1}$-functions from irregular domains, proceedings of Complex Analysis and Dynamical Systems VI. Part 1, 177-189, Contemp. Math. 653, Amer. Math. Soc., Providence, RI, 2015.

[11] P. Harjulehto, R. Hurri-Syrjänen, and J. Kapulainen, An embedding into an Orlicz space for irregular John domains, Comput. Methods Funct. Theory 14 (2014), 257-277.

[12] P. Harjulehto, R. Hurri-Syrjänen, and A. V. Vähäkangas, On the $(1, p)$ Poincaré inequality, Illinois J. Math. 56 (2012), 905-930.

[13] L. I. Hedberg, On certain convolution inequalities, Proc. Amer. Math. Soc. 36 (1972), 505-510.

[14] T. Kilpeläinen and J. Maly, Sobolev inequalities on sets with irregular boundaries, Z. Anal. Angew. 19 (2000), 369-380.

[15] V. Kokilashvili and M. Krbec, Weighted Inequalities in Lorentz and Orlicz Spaces. World Scientific. Singapore, 1991.

[16] F-Y. Maeda, Y. Mizuta, T. Ohno and T. Shimomura: Boundedness of maximal operators and Sobolev's inequality on Musielak-Orlicz-Morrey spaces, Bull. Sci. Math. 137 (2013), no. 1, 76-96. 
[17] V. Maz'ya, Sobolev Spaces with Applications to Elliptic Partial Differential Equations, 2nd revised and augmented Edition, A Series of Comprehensive Studies in Mathematics, 342, Springer Heidelberg Dordrecht London New York, 2011.

[18] V. Maz'ya and S. Poborchi, Differentiable Functions on Bad Domains, World Scientific, Singapore, 1997.

[19] J. Moser, A sharp form of an inequality of N. Trudinger's inequality, Indiana Univ. Math. J. 11 (1971), 1077-1092.

[20] T. Ohno and T. Shimomura, Trudinger's inequality for Riesz potentials of functions in Musielak-Orlicz spaces, Bull. Sci. Math. 138 (2014), 225-235.

[21] R. O'Neil, Fractional integration in Orlicz spaces, Trans. Amer. Math. Soc. 115 (1965), 300-328.

[22] J. Peetre, Espaces d'interpolation et théorème de Soboleff, Ann. Inst. Fourier (Grenoble) 16 (1966), 279-317.

[23] S. I. Pohozhaev, On the imbedding Sobolev theorem for $p l=n$, Doklady Conference, Section Math. Moscow Power Inst.(1965), 158-170 (Russian).

[24] Yu. G. Reshetnyak, Integral representations of differentiable functions in domains with nonsmooth boundary (Russian), Sibirsk. Mat. Zh. 21(1980), 108116; translation in Sib. Math. J.21 (1981), 833-839.

[25] E. M. Stein, Singular Integrals and Differentiability Properties of Functions Princeton Univ. Press, Princeton, New Jersey, 1970.

[26] R. S. Strichartz, A note on Trudinger's extension of Sobolev's inequality, Indiana Univ. Math. J. 21 (1972), 841-842.

[27] A. Torchinsky, Interpolation of operators and Orlicz classes, Studia Math. 59 (1976), 177-207.

[28] N. S. Trudinger, On imbeddings into Orlicz spaces and some applications, J. Math. Mech. 17 (1967), 473-483.

[29] V. I. Yudovich, On some estimates connected with integral operators and with solutions of elliptic equations (Russian), Dokl. Akad. Nauk SSSR 138 (1961), 805-808; translation in Sov. Math. Dokl. 2 (1961), 746-749.

(Petteri Harjulehto) Department of Mathematics and Statistics, FI20014 University of Turku, Finland

E-mail address: petteri.harjulehto@utu.fi

(Ritva Hurri-Syrjänen) Department of Mathematics and Statistics, FI00014 University of Helsinki, Finland

E-mail address: ritva.hurri-syrjanen@helsinki.fi 